\title{
The Developmental Behaviour Checklist profile in young children on the autism spectrum: The impact of child and family factors
}

Dawn Adams ${ }^{1,2} \mathrm{PhD}$, Jessica Paynter ${ }^{2,3} \mathrm{PhD}$, Megan Clark ${ }^{1,2}$ PhD, Jacqueline Roberts ${ }^{1,2} \mathrm{PhD}$, \& Deb Keen ${ }^{1,2} \mathrm{PhD}$

${ }^{1}$ Autism Centre of Excellence, School of Education and Professional Studies, and Griffith Institute for Educational Research, Griffith University, Australia

${ }^{2}$ Cooperative Research Centre for Living with Autism (Autism CRC), Long Pocket, Brisbane, Queensland, Australia

${ }^{3}$ School of Applied Psychology and Menzies Health Institute Queensland Griffith University, Australia

Running Head: Behavior in young children with autism

\section{Acknowledgements}

We are grateful to the children with autism and their families for giving their time to support this research study. The authors acknowledge the work of the LASA team members: Susan Bruck, Trevor Clark, Sandra Devaraj, Robyn Garland, Honey Heussler, Antoinette Hodge, Patricia Howlin, Kate Simpson, Natalie Silove, David Trembath, Madonna Tucker, Marleen Westerveld, and Katrina Williams.

\section{Funding statement and declaration of conflicting interests}

The authors acknowledge the financial support of the Cooperative Research Centre for Living with Autism (Autism CRC), established and supported under the Australian Government's Cooperative Research Centres Program (project 2.007).

Dawn Adams declares that she has no conflict of interest with respect to this publication. Jessica Paynter declares that she has no conflict of interest with respect to this publication. Megan Clark declares that she has no conflict of interest with respect to this publication. Jacqueline Roberts declares that she has no conflict of interest with respect to this publication. Deb Keen declares that she has no conflict of interest with respect to this publication 


\begin{abstract}
High levels of emotional/behavioural difficulties are frequently reported in children on the autism spectrum. However, given the diversity in profiles, there is a need to explore such behaviours in relation to individual factors. Parents of 130 children aged 4-5 on the autism spectrum completed measures of behaviour and adaptive behaviour. Hierarchical multiple regressions explored child and family characteristics in relation to children's emotional/behavioural presentation. Different aspects of the behavioural profile were associated with different factors, with child autism characteristics, medication use and parent mental health making significant unique contributions to a range of behavioural subscales. Understanding individual profiles beyond total scores is therefore needed to truly understand the emotional and behavioural profile of specific subgroups.
\end{abstract}




\section{The Developmental Behaviour Checklist (DBC) profile in young children on the autism spectrum: The impact of child and family factors}

Challenging and/or emotional behaviours are reported to be more common in children on the autism spectrum than in typically developing children or those with other disabilities including intellectual disability (ID) (Brereton, Tonge, \& Einfeld, 2006; Mayes, Calhoun, Murray, Ahuja, \& Smith, 2011). Within 4-year-olds on the autism spectrum, Soke, Maenner, Christensen, Kurzius-Spencer, and Schieve (2018) report that $24.7 \%$ displayed self-injurious behaviour, $41 \%$ aggression, and $56.2 \%$ had a mood disorder. Identification and understanding of such behaviours is important as they can mask or modify the autism profile, impact on the diagnostic process or accuracy, increase the need for services, and reduce the quality of life for the individual on the spectrum and their family members (Adams, Handley, et al., 2018; Close, Lee, Kaufmann, \& Zimmerman, 2012; Sikora, Vora, Coury, \& Rosenberg, 2012; Vohra, Madhavan, \& Sambamoorthi, 2016). Kurzius-Spencer et al. (2018) compared challenging behaviour in a large sample of 8 -year-old children on the autism spectrum with co-occurring ID (ASD+ID; $n=3,295)$ and without ID (ASD-ID only; $n=6,083)$. The majority of children $(60 \%)$ had six or more co-occurring challenging behaviours, regardless of co-occurring ID, although the risk of behaviours was different for children depending on IQ. The ASD-ID group were more likely to experience mood abnormalities and sleep disturbances, and the ASD+ID group were more likely to show self-injurious behaviour, unusual fears, and eating problems. This highlights the need to explore behavioural presentation not only by diagnosis, but also by child factors, including ability. 
Population-based studies can help to identifying factors that are important in understanding behavioural presentation of children on the autism spectrum. Using UK population-based data, Totsika et al. (2011) found that both child factors (age, gender, autism diagnostic status, presence of ID) and family factors (family socio-economic status, maternal emotional disorder) predicted elevated emotional and behavioural problems, with autism symptomatology and ID making the two strongest contributions to behavioural presentation. This is not a unique finding, with other studies also reporting associations between challenging behaviour and autism characteristics (see Baghdadli, Pascal, Grisi \& Aussilloux, 2003), child gender (see meta-analysis of by McClintock, Hall \& Oliver 2003) and child age (Maskey et al. 2013). It is also important to consider other transient factors which may influence behavioural presentation, such as child medication. Children who are prescribed medications have significantly higher rates of behavioural and/or emotional difficulties (Tureck, Matson, Turygin \& Macmillian, 2013) some of which are noted to be side-effects of the medications (see review by Taylor, 2016). Whilst it is not possible for studies to control for every factor that may impact upon emotional or behavioural presentations simulatanously, there is a clear need for studies to begin to explore the impact of multiple child and family factors on different aspects of the behavioural and emotional profile of children on the autism spectrum.

A range of measures exist to assess the extent of challenging and emotional behaviours in children, such as the Child Behaviour Checklist (Achenbach \& Edlbrock, 1983), Behaviour Assessment Scale for Children (Reynolds \& Kamphaus, 2015), and the Strengths and Difficulties Questionnaire (Goodman, 1997). However, few are specific to children with developmental disabilities or autism. This is problematic, as measures normed and developed for typically developing children or 
other clinical groups may not be sensitive to the needs of children on the autism spectrum. For example, these measures may not discriminate between whether behaviours (e.g., social avoidance) are associated with the profile of autism or represent additional challenges. Given that levels of behaviours that challenge are higher in children on the autism spectrum, such measures may not be sensitive to variations in severity, with the potential for ceiling effects, highlighting the need for appropriate clinical norms. Finally, measures developed for typically developing children may not include behaviours common in autism but rarely seen in typical development, such as pica, echolalia, or social relating problems (Einfeld \& Tonge, 1995). To address this need for a measure for children with a range of developmental disabilities, the Developmental Behaviour Checklist (DBC) was developed from clinical descriptions of behavioural and emotional symptoms of children and adolescents with ID including those with co-occurring diagnoses on the autism spectrum (Einfeld \& Tonge, 1995).

Although the DBC was not developed specifically for measuring behaviour within individuals on the autism spectrum, children on the autism spectrum were included in the normative samples and studies of psychometrics in the DBC manual (Einfeld \& Tonge, 1995). The DBC has been used in research with children on the autism spectrum with a range of ability levels as a measure of severity of challenging behaviours, a predictor of parent well-being (e.g., Herring et al., 2006; Jellett, Wood, Giallo, \& Seymour, 2015), and an outcome measure in intervention studies (e.g., Tonge et al., 2006; Wright et al., 2011). However, only a small number of studies have specifically explored the profiles of children on the autism spectrum on this measure. 
Brereton et al. (2006) compared DBC-P ratings for people with a diagnosis on the autism spectrum $(n=367)$ or ID without a diagnosis of autism $(n=550)$ ranging from approximately 4 years of age through to adults. When age, gender, and IQ were controlled for, those with a diagnosis on the autism spectrum scored significantly higher on all subscales and algorithms (except the antisocial subscale) than those with an ID diagnosis. Further analyses investigated the impact of cognitive level for those with a diagnosis on the autism spectrum by comparing scores across DSM-IV categories of normal, borderline, mild, moderate, and severe ID, which revealed differences by IQ. Children on the autism spectrum with a moderate-severe ID had higher scores on the self-absorbed subscale than other participant groups, and a higher score on the social relating subscale than those with borderline-normal IQ. Those with borderline-normal IQ showed greater problems with communication disturbance than those with moderate to severe ID, perhaps reflecting more limited communication skills in those with a diagnosis of ID. This therefore suggests that behavioural and/or emotional difficulties may be present across the ability range, and therefore more research is needed in order to further explore and identify the factors associated with these different presentations.

The DBC profiles of children on the autism spectrum aged $4-8$ years were explored by Chandler et al. (2016). Parents $(n=277)$ and teachers $(n=228)$ completed the DBC-P and DBC-T respectively, and a subset of parents also completed a follow-up DBC-P $(n=93)$. The mean IQ for the sample was 72.7 , with $35 \%$ obtaining an IQ below 70 . Overall, $79 \%$ of the participants scored above the clinical cut-off on the DBC-P and 62\% scored above the clinical cut-off on the DBCT total problem behaviour score (TBPS). Despite a lack of association between DBCP TBPS and IQ, significant differences were found on subscales between those with 
an IQ above and below 70. Those with an IQ over 70 scored higher on DBC-P disruptive/antisocial, depression, and anxious behaviour subscales, while those with an IQ under 70 scored higher on the self-absorbed and hyperactivity scales. No correlations were found between DBC-P TBPS and age, IQ, receptive language, gender, parental education, or ethnicity. However, a higher DBC-P TBPS was associated with a greater autism of autism characteristics on the Social Communication Questionnaire (SCQ; Rutter, Bailey, \& Lord, 2003), higher deprivation index, parental unemployment, and more children in the home, highlighting the need to explore both child and family factors alongside child level of ability.

Classifying sub-groups of children on the autism spectrum based on IQ while investigating behaviour, as completed in the Chandler et al. (2016) study, can be useful, but it could be argued that splitting scores above and below a cut-off of 70 limits the information that can be gathered regarding the relationship between a range of IQ scores and emotional and behavioural presentation. Chandler et al. correlate IQ with the DBC-P TBPS, but do not explore the relationship between IQ and DBC-P subscales and did not enter the IQ scores into the regression analyses for DBC-P TBPS, limiting the extent to which DBC-P scores could be investigated in relation to other independent variables including child ability. In a commentary on Chandler et al.'s article, Smith (2016) argued for the importance of exploring behaviour based upon an adaptive behaviour measure, rather than IQ. This is particularly important as although many studies report a correlation between IQ and adaptive behaviour in children on the spectrum (e.g. Kenworth, Case, Harms, Martin \& Wallace, 2010; Liss et al., 2001), this is not a consistent finding. For example, those with an IQ score of lower than 70 have been reported to show relative strengths in their adaptive 
functioning, with the reverse found for those with IQ scores above 70 (e.g., Yang, Paynter \& Gilmore, 2015). In addition, correlation between two measures implies that scores show a statistical relationship, but it does not mean that the two measures describe the child as functioning at the same level.

There are questions around the validity of the results of IQ assessments in children on the autism spectrum (Nader, Courchesne, Dawson, \& Soulieres, 2016) and there is increasing recognition that factors often associated with autism, including challenging behaviour itself, can influence IQ assessment outcomes (Akshoomoff, 2006). While adaptive behaviour is often considered the best indicator of level of functioning, rather than IQ, independent from autism symptoms (Klin et al., 2007), to date, studies have not tended to use adaptive behaviour as an independent variable to determine if patterns emerge according to different overall levels of adaptive functioning for children on the autism spectrum. The limited studies that have explored relationships between adaptive behaviour and behavioural profiles have either used a measure of behaviour as a dependent variable to explore language outcomes on a measure of adaptive behaviour (e.g. Park, Yelland, Taffe \& Gray, 2012) or only used one specific subscale of adaptive behaviour as an independent variable to explore behaviour (e.g. Williams, Siegel \& Mazefsky, 2018).

\section{The current study}

The current study was therefore designed to extend upon the work of Chandler et al. (2016) while addressing the commentary by Smith (2016) by investigating whether scores on multiple domains of adaptive behaviour added any additional explanation of variance over and above other child and family factors which have previously been established as predictive behavioural and/or emotional profiles of children on the spectrum. The focus on $4-5$ years was considered important as this is 
a key developmental period and critical time for early intervention and prevention of future challenging behaviour. To achieve this, the following research questions were posed:

1. To what degree do child and family factors and adaptive functioning predict behavioural and emotional presentation in $4-5$-year-old children on the autism spectrum?

2. If so, do these prediction models differ dependent upon the aspect of behavioural and emotional presentation that is being assessed?

Given the work of Chandler et al. (2016), the following hypotheses were made:

1. Child adaptive behaviour scores will have a significant impact upon DBC-P total and subscale scores.

2. When controlling for child factors (e.g., SCQ, medication) and family factors, child ability (i.e., adaptive behaviour scores) will have an independent and significant impact upon DBC-P total and subscale scores.

3. The impact of child and family factors and child adaptive behaviour will differ across the DBC-P subscale scores.

\section{Method}

This study used data collected at the first time point of the $<$ removed for blind review $>$, an ongoing sequential study exploring educational and social outcomes for children on the autism spectrum. Ethical approval for this study was obtained from all participating universities and health authorities. Informed consent was obtained from all individual participants included in the study.

\section{Recruitment Procedures}

The full recruitment procedure for the $<$ removed for blind review $>$ study is described in the published protocol $<$ removed for blind review $>$. In brief, parents of 
children on the autism spectrum aged $4-5$ or $9-10$ from across Australia were informed of the study through community service providers and education and health services. If parents met the two inclusion criteria (the child had a confirmed diagnosis of being on the autism spectrum and the child was aged $4-5$ or $9-10$ at time of enrolment) they completed an online enrolment form and were then invited to complete a range of questionnaires. The sample was therefore self-selecting and, for reasons of confidentiality, the research team was not provided with any details on children or families who received the information but did not enrol into the study. As this study aims to explore the profile of scores on the DBC-P in young children on the autism spectrum, only children within the younger cohort, i.e, those aged 4-5 years at time of enrolment, were included in this study.

Diagnosis was confirmed by the provision of community diagnostic reports as well as a parent-completed screener of autism symptomatology (SCQ; Rutter et al., 2003). Two participants were excluded from this study as their SCQ score was below the cut-off of 11 for autism screening on the SCQ (Lee, David, Rusyniak, Landa, \& Newschaffer, 2007) and the community diagnostic reports were not based upon goldstandard assessment methods. Almost all (96.9\%) children were diagnosed by a paediatrician, with the remaining diagnoses being made or confirmed by professionals from multiple disciplines including psychologists and speech and language pathologists.

\section{Participants}

The final sample consisted of 130 children with a diagnosis on the autism spectrum who were age $4-5$ at time of enrolment. Participant characteristics are described in Table 1 . The sample was predominantly male (83\%) and attended general education/mainstream schools (with or without support). Almost half (45.3\%) 
had at least one additional diagnosis or medical condition, the most common of which was reported to be an attentional disorder (ADHD/ADD; $10 \%)$ and asthma (6.2\%). All informants, who were predominantly mothers (88.5\%), lived within the family home and with the child full-time. The majority of respondents had a tertiary education, with a family income of AUD\$80,000 or above. For reference, the 2016 Australian Census noted that $30.9 \%$ of the population had studied for and achieved educational qualifications of diploma (i.e., vocational training post-school qualification) or above and the median annual family income was AUD\$90,168.

Table 1.

Participant Characteristics Based Upon Parent Report $(N=130)$

\begin{tabular}{|c|c|c|c|}
\hline & & $\mathrm{N}$ & $\%$ \\
\hline \multicolumn{4}{|c|}{ Mean age of child: 60.2 months ( $\mathrm{SD}=6$ months) } \\
\hline \multirow{4}{*}{$\begin{array}{l}\text { Vineland Adaptive } \\
\text { Behaviour Scales } \\
\text { standard score below } 70\end{array}$} & 0 domains & 50 & 38.5 \\
\hline & 1 domain & 29 & 22.3 \\
\hline & 2 domains & 19 & 14.6 \\
\hline & 3 domains & 32 & 24.6 \\
\hline \multicolumn{2}{|c|}{$\begin{array}{l}\text { Additional diagnosis or medical condition (e.g., ADHD, } \\
\text { asthma, coordination difficulties) }\end{array}$} & 59 & 45.3 \\
\hline \multirow[t]{4}{*}{ Prescribed medications } & Melatonin & 17 & 13.1 \\
\hline & ADHD medication & 9 & 6.9 \\
\hline & Rispiridal/risperidone & 7 & 5.4 \\
\hline & Anticonvulsants & 4 & 3.1 \\
\hline \multicolumn{2}{|c|}{ Informant education tertiary education or above } & 105 & 81 \\
\hline \multicolumn{2}{|c|}{ Household income of $\$ 80,000$ or above } & 76 & 59.2 \\
\hline \multirow{3}{*}{$\begin{array}{l}\text { Informant main } \\
\text { occupation }\end{array}$} & Employed & 71 & 54.6 \\
\hline & Full-time carer & 40 & 31 \\
\hline & Studying & 13 & 12.3 \\
\hline Child education setting & Mainstream preschool/school & 19 & 14.6 \\
\hline
\end{tabular}




\section{Measures}

Demographic characteristics. These were collected by a parent questionnaire focusing upon child (age, gender, age at diagnosis, additional diagnoses and medication), parent (education, employment, mental health status), and household (income) variables. For the purpose of the analysis, child medication and parent mental health status were coded into a dichotomous variable of yes (taking prescribed medication/stated that they have a diagnosis of a mental health condition) or no (was not taking prescribed medication/stated that they did not have a diagnosis of a mental health condition).

Autism characteristics. The SCQ (Rutter et al., 2003) is a behavioural checklist that requires parents to indicate the presence of certain social, communicative, or stereotyped behaviours by answering yes or no to 40 items. The SCQ has been extensively researched, with a recent meta-analysis (Chesnut, Wei, Barnard-Brak, \& Richman, 2017) concluding that it is an acceptable screening measure for autism spectrum disorder (area under the receiver operating characteristic curve .89). Although not originally designed for this purpose, as a higher score represents a higher number of behaviours, which may be considered indicative of autism, within this study the child's SCQ score was used as a proxy measure of autism characteristics. Cronbach's alpha for this sample was .91.

Behavioural and emotional presentation. The Developmental Behaviour Checklist Primary Carer Version (DBC-P) (Einfeld \& Tonge, 1995) was used to evaluate behavioural and emotional symptoms. This is a measure of behaviour and 
emotional problems in children aged $4-18$ years. For each item, parents rate whether the statement is not true (0), somewhat or sometimes true (1), or very/often true (2) of their child. A total behaviour problem score (TBPS; ranging from $0-192$ ) is obtained from the sum of all items, with a score of at least 46 recommended for identifying clinically significant behavioural or emotional problems. The DBC has five subscales: disruptive/antisocial (e.g., manipulates, abusive, tantrums, hits), self-absorbed (e.g., eats non-food, preoccupied with trivial items, hums, grunts), communication disturbance (e.g., echolalia, perseveration, talks to self), anxiety (e.g., separation anxiety, distressed if alone, phobias, cries easily) and social-relating (e.g., doesn't show affection, resists cuddling, aloof, doesn't respond to others' feelings). There are also additional scales that can be used to identify specific psychiatric syndromes, including the Hyperactivity scale and the Depression subscale which are included within this study. The internal consistency, inter-rater and test-retest reliability, and concurrent validity are stated to be satisfactory or above in children with ID (Einfeld \& Tonge, 1995). For this sample, the Cronbach's alpha ranged from acceptable (communication disturbance, $\alpha=.71$; for the anxious behaviour rating scale, $\alpha=.79$; and social-relating, $\alpha=.71$ ) to excellent (TBPS, $\alpha=.96$; disruptive/antisocial, $\alpha=.91$; for self-absorbed, $\alpha=.89$ ).

Adaptive behaviour. The Vineland Adaptive Behavior Scales $2^{\text {nd }}$ Edition (VABS-II; Sparrow, Cicchetti, \& Balla, 2005) was used to evaluate adaptive behaviour skills. This gathers information from the informant on an individual's daily functional skills and adaptive behaviour. This study collected information from three domains: socialisation, daily living skills, and communication. Standard scores were used for analysis to allow comparisons with the Chandler et al. (2016) study. In order to maintain consistency with data collection across the 6-year study, the motor skills 
domain was not administered. The VABS-II manual reports good test-retest reliability, with correlations ranging from $.80-.95$ and inter-rater reliability correlations ranging from $.75-.85$.

\section{Data Analysis}

All analysis were undertaken on SPSS version 25. Prior to examining prediction models, bivariate Pearson and point-biserial correlation analyses for participants within the regression models were conducted. These revealed no collinearity of predictors $(r>.80)$, thus all were retained (see Table 2).

Table 2.

Pearson Correlations Between Predictor Variables

\begin{tabular}{|c|c|c|c|c|c|c|c|c|}
\hline & 2 & 3 & 4 & 5 & 6 & 7 & 8 & 9 \\
\hline 1. VABS DLS & $.67 * *$ & $.60 * *$ & .05 & .11 & $-.34 * *$ & -.13 & .19 & .10 \\
\hline 2. VABS Com. & - & $.68 * *$ & -.01 & -.20 & $-.40 * *$ & -.06 & $.29 *$ & .08 \\
\hline 3. VABS Soc. & & - & -.14 & 0.20 & $-.46^{* *}$ & -.18 & $.29 *$ & .11 \\
\hline 4. Age & & & - & -.09 & .11 & .02 & -.14 & .13 \\
\hline 5. Gender ${ }^{\mathrm{a}}$ & & & & - & .10 & .05 & -.07 & .03 \\
\hline 6. SCQ & & & & & - & .12 & -.22 & -.01 \\
\hline 7. Child Medication ${ }^{\mathrm{a}}$ & & & & & & - & -.09 & .03 \\
\hline 8. Income & & & & & & & - & -.10 \\
\hline 9. Parent $\mathrm{MH}^{\mathrm{a}}$ & & & & & & & & - \\
\hline
\end{tabular}

To explore the relationships between child factors, child adaptive behaviour and family factors and the scores from the DBC-P, Pearsons and Pointserial correlations were undertaken. Following this, Hierarchical multiple regression (HMR) analyses, with each DBC-P total or subscale score as the outcome variable, were 
conducted to investigate the variance explained by the combination of variables at each step, alongside each variable's unique contributions. To ensure thorough exploration of the impact of adaptive behaviour alongside child and family factors, all predictor variables were included per regression model. Variables were entered in three steps. Child factors previously shown to influence child behavioural presentation (age, gender, SCQ total score, and no/yes as to whether they were taking prescribed medication) were entered first. At step two, family factors previously shown to influence child behavioural presentation (family income, and no/yes as to whether the mother had disclosed that they have a diagnosis of a mental health condition) were entered. In order to see if child adaptive behaviour explained additional variance over and above that explained by established child and family factors (entered in step 1 and 2), Vineland standardised domain scores were entered at step three. All steps of the regression were undertaken using the SPSS “enter" procedure (forced entry), where all variables within each step were entered simultaneously. This is considered an appropriate analysis when dealing with a small set of predictors and it is not known which independent variables will create the best prediction equation. For dichotomous no/yes variables, no was coded as 0 and yes as 1. Standardised coefficients $(\beta)$ are reported for ease of interpretation. The contribution of significant variables $\left(\mathrm{sr}^{2}\right)$ was calculated by squaring the semi-partial correlation coefficient of the variable.

Although the design required multiple analyses which potentially raised the possibility of increased familywise error, Bonferroni correction was considered too conservative (Perneger, 1998) as increased risk of Type 1 errors was considered less of a concern that Type 2 errors. Thus, a $p$-value of .01 was set for all analyses.

\section{Results}




\section{Adaptive Behaviour}

As shown in Table 1, 32 (24.6\%) of the participants had scores below 70 on all three domains of the VABS and $50(38.5 \%)$ had scores above 70 on all three domains of the VABS. The remaining $36.9 \%$ of participants had a mix of scores above and below 70 .

\section{DBC-P Profile}

Overall, $79 \%$ of children met the clinical cut-off on the DBC-P for significant emotional and/or behavioural problems. The mean score for the DBC-P TBPS and subscale scores are presented in Table 3.

\section{Relationships between DBC-P TBPS and subscales and child and family factors}

In order to explore the relationships between the DBC-P TBPS and subscale scores and the child factors (age, gender, SCQ, medication status), family factors (family income, parent mental health diagnosis) and the child adaptive behaviour standardised scores, Pearsons and Pointserial correlations were undertaken. The results of these are reported in Table 3. Child SCQ score was positively correlated with all DBC-P scores whereas child age and gender were not correlated with any DBC-P scores. Child medication status (no/yes taking prescribed medication) was positively correlated with five of the eight DBC-P scores. The child adaptive behaviour standardised scores were all negatively correlated with the DBC-P selfabsorbed subscale score, suggesting that those with higher adaptive behaviour scores have lower scores on the self-absorbed subscale score (see measures section for example items/behaviours within this subscale). 
Table 3

DBC-P Total and subscale sample mean (SD) and Pearsons or Pointserial correlations coefficients with child and family factors

\begin{tabular}{|c|c|c|c|c|c|c|c|c|c|c|}
\hline \multirow[b]{2}{*}{ DBC-P Subscale } & \multirow{2}{*}{$\begin{array}{c}\text { Sample } \\
\text { Mean } \\
(S D)\end{array}$} & \multicolumn{4}{|c|}{ Child factors } & \multicolumn{2}{|c|}{ Family factors } & \multicolumn{3}{|c|}{$\begin{array}{c}\text { Child Adaptive Behaviour } \\
\text { (Standard score) }\end{array}$} \\
\hline & & $\begin{array}{c}\text { Age } \\
\text { (months) }\end{array}$ & Gender & $\begin{array}{l}\text { SCQ } \\
\text { Total } \\
\text { Score }\end{array}$ & $\begin{array}{l}\text { Child } \\
\text { Medication }\end{array}$ & $\begin{array}{l}\text { Family } \\
\text { Income }\end{array}$ & $\begin{array}{c}\text { Parent } \\
\text { Mental } \\
\text { health } \\
\text { diagnosis }\end{array}$ & $\begin{array}{l}\text { VABS } \\
\text { Daily } \\
\text { Living } \\
\text { Skills }\end{array}$ & $\begin{array}{c}\text { VABS } \\
\text { Communication }\end{array}$ & $\begin{array}{c}\text { VABS } \\
\text { Socialisation }\end{array}$ \\
\hline TPBS & $\begin{array}{c}69.9 \\
(27.7)\end{array}$ & -.03 & .05 & $.54 * *$ & $.30^{*}$ & $-.25^{*}$ & -.20 & -.10 & -.20 & -.23 \\
\hline $\begin{array}{l}\text { Disruptive } \\
\text { Behaviour }\end{array}$ & $\begin{array}{c}20.0 \\
(10.2)\end{array}$ & .03 & .02 & $.28 *$ & $.23^{*}$ & $-.24 *$ & $-.33^{* *}$ & .01 & -.04 & -.09 \\
\hline Self-absorbed & $\begin{array}{c}23.0 \\
(10.4)\end{array}$ & -.11 & .15 & $.58 * *$ & .21 & -.19 & -.05 & $-.33 * *$ & $-.43 * *$ & $-.39 * *$ \\
\hline $\begin{array}{l}\text { Communication } \\
\text { disturbance }\end{array}$ & $\begin{array}{c}9.8 \\
(4.4)\end{array}$ & -.05 & -.07 & $.45^{* *}$ & $.23 *$ & -.05 & -.04 & .07 & .01 & -.07 \\
\hline Anxiety & $\begin{array}{c}8.0 \\
(4.1)\end{array}$ & -.02 & -.05 & $.39 * *$ & .31 & -.20 & .15 & .05 & .01 & -.04 \\
\hline Social Relating & $\begin{array}{c}6.0 \\
(3.1)\end{array}$ & -.02 & .04 & $.53 * *$ & .19 & $-.31 *$ & -.17 & -.03 & -.14 & $-.28 *$ \\
\hline Hyperactivity & $\begin{array}{c}8.0 \\
(3.0)\end{array}$ & .04 & 0 & $.28 *$ & $.24 *$ & -.22 & -.11 & -.15 & -.19 & $-.27 *$ \\
\hline Depression & $\begin{array}{c}5.7 \\
(3.7)\end{array}$ & .08 & -.02 & $-.32 * *$ & $.28^{*}$ & -.19 & $-.33 * *$ & .02 & -.04 & -.06 \\
\hline
\end{tabular}




\section{HMR of child and family factors to DBC-P scores}

Using the DBC-P total and subscale scores as the criterion variables, a series of HMR were undertaken using the predictor entry strategy described in the data analysis section. The results, including the standardised Beta values for each variable in each step of the model, are summarised in Table 4.

For the DBC-P TBPS, the baseline model (including child age, gender, SCQ score, and medication) was significant and accounted for $37 \%$ of the variance in children's emotional and behavioural presentation $(F(4,110)=15.78, p<.001)$. Child SCQ score $(\beta=-.51)$ and medication status $(\beta=.29)$ were identified as unique significant predictors explaining $24 \%$ and $9 \%$ of the variance respectively. The second stage model, which included child age, gender, SCQ score, and whether or not the child was taking prescribed medication (yes/no) and two family factors: family income and whether or not the parent disclosed that they have a diagnosis of a mental health condition was significant $(F(6,110)=13.21, p<.001)$ and accounted for an additional and significant $6 \%$ of the variance from the baseline model $\left(F_{\text {change }}(2,108)\right.$ $=5.49, p=.005)$. SCQ score $(\beta=.53)$, medication status $(\beta=.30)$, and parental mental health status $(\beta=.20)$ were identified as unique significant predictors explaining $23 \%, 8 \%$, and $5 \%$ of the variance respectively. The results of the secondstage HMR indicated that higher SCQ scores, the child taking prescribed medication, and the parent reporting having a mental health diagnosis predicted higher scores on the TBPS of the DBC-P. The third stage model (including the three VABS daily living skills, communication, and socialisation standard scores, child age, gender, SCQ score, medication use, family income, and parental mental health diagnosis) was significant $(F(9,105)=8.85, p<.001)$ but did not account for any additional variance from the second stage model $\left(F_{\text {change }}(3,105)=.52, p=.66\right)$. 
For the DBC-P Disruptive Behaviour subscale, the baseline model was significant, explaining $15 \%$ of the variance in disruptive behaviours $(F(4,110)=4.68$, $p=.002)$. Child SCQ score $(\beta=.23)$ and medication status $(\beta=.29)$ were significant unique predictors explaining $5 \%$ and $9 \%$ of the variance respectively. The second stage model was also significant $(F(6,108)=6.51, p<.001)$, and accounted for an additional and significant $12 \%$ of the variance $\left(F_{\text {change }}(2,108)=8.85, \mathrm{p}<.001\right)$. SCQ score $(\beta=.23)$, medication status $(\beta=.28)$, and parental mental health $(\beta=.33)$ were identified as unique significant predictors explaining $5 \%, 8 \%$, and $10 \%$ of the variance respectively. The third stage model was also significant $(F(9,105)=4.59$, $\mathrm{p}<.001)$ but did not account for an additional and significant variance from the second stage model $\left(F_{\text {change }}(3,105)=.81, p=.49\right)$. Child SCQ score $(\beta=.28)$, medication status $(\beta=.28)$, and parental mental health status $(\beta=.31)$ remained the unique significant predictors explaining $6 \%, 7 \%$, and $9 \%$ of the variance respectively. The results of the third-stage HMR indicated that higher SCQ scores, the child taking prescribed medication, and the parent reporting having a mental health diagnosis predicted higher scores on the Disruptive Behaviour subscale.

For the DBC-P Self-absorbed subscale, the baseline model was significant and accounted for $39 \%$ of the variance $(F(4,110)=17.84, p<.001)$. Child SCQ score $(\beta$ $.54)$, medication status $(\beta=.22)$, and age $(\beta=-.22)$ were identified as significant unique predictors, explaining $28 \%, 5 \%$ and $4 \%$ of the variance respectively. The second stage model was also significant $(F(6,108)=12.21, p<.001)$ but did not contribute any additional and significant variance from the baseline model $\left(F_{\text {change }}\right.$ $(2,108)=.97, p=.38)$ and did not substantively change any coefficients for variables in block 1 . The third stage model was significant $(F(9,105)=9.74, p<.001)$ but did not add a significant amount of variance from the second stage model $\left(F_{\text {change }}(3,105)\right.$ 
$=3.26, p=.02)$. Child SCQ score $(\beta=.54)$, medication status $(\beta=.22)$, and child chronological age $(\beta=-.20)$ remained unique significant predictors explaining $15 \%$, $5 \%$, and $4 \%$ of the variance respectively. The results of the third-stage HMR indicated that higher SCQ scores, the child taking prescribed medication, and a younger chronological age predicted higher scores on the Self-absorbed subscale.

For the DBC-P Communication Disturbance subscale, the baseline model was significant and explained $24 \%$ of the variance $(F(4,110)=9.04, p<.001)$. Child SCQ score $(\beta=.46)$ was the only signicant unique predictor explaining $20 \%$ of the variance. The second stage model was also significant $(F(6,108)=6.11, p<.001)$ but did not account for significantly additional variance $\left(F_{\text {change }}(2,108)=.45, p=.64\right)$ and did not substantively change any coefficients for variables in block 1 . The third stage model was also significant $(F(9,105)=5.17, p<.001)$ but did not add any significant amount of variance from the second stage model $\left(F_{\text {change }}(3,105)=2.69, p=.05\right)$ and did not change which predictor variables reach significance, with child SCQ score ( $\beta$ $=.58$ ) remaining the only significant unique predictor accounting for $25 \%$ of the variance.

For the DBC-P Anxiety subscale, the baseline model was significant and explained $20 \%$ of the variance $(F(4,110)=7.03, p<.001)$. Child SCQ score $(\beta=.39)$ was the only unique significant predictor identified explaining $14 \%$ of the variance. The second stage model was significant $(F(6,108)=5.53, p<.001)$ but did not account for any additional and significant variance $\left(F_{\text {change }}(2,108)=2.21, p=.11\right)$ and did not substantively change any coefficients for variables in block 1 . The third stage model was also significant $(F(9,105)=4.78, p<.001)$ but did not add any significant amount of variance from the second stage model $\left(F_{\text {change }}(3,105)=2.75, p=.04\right)$. The significant predictors within the third stage model were child SCQ score $(\beta=.49)$ and 
and prescribed medication $(\beta=.22)$, explaining $19 \%$ and $5 \%$ of the variance respectively.

For the DBC-P Social Relating subscale, the baseline model was significant and accounted for $32 \%$ of the variance $(F(4,110)=12.70, p<.001)$. Child SCQ score $(\beta=.51)$ was the only significant predictor explaining $25 \%$ of the variance. The second stage model was also significant $(F(6,108)=11.38, p<.001)$ and accounted for an additional and significant $7 \%$ of the variance $\left(F_{\text {change }}(2,108)=6.29, p=.003\right)$. Again, child SCQ score $(\beta=.49)$ was the only significant predictor, explaining $22 \%$ of the variance. The third stage model was significant $(F(9,105)=8.09, p<.001)$ but did not account for significant additional variance from the second stage model $\left(F_{\text {change }}(3,105)=1.32, p=.27\right)$. The two significant predictors within the third stage model were child SCQ score $(\beta=.50)$ and child age $(\beta=-.21)$, with these two variables explaining $19 \%$ and $3 \%$ of the variance respectively).

For the DBC-P Hyperactivity subscale, the baseline model was not significant $(F(4,109)=3.482 .54, p=.10)$. However, the second stage model $(F(6,107)=3.10, p=$ .008 ) was significant and explained $15 \%$ of the variance, although none of the variables were unique significant predictors. The third stage model, which added in VABS scores into the regression model, was not significant, $F(9,104)=2.25, p=.02$.

For the DBC-P Depression subscale, the baseline model was significant and accounted for $19 \%$ of the variance $(F(4,110)=6.41, p<.001)$. Child SCQ total score $(\beta=.27)$ and medication status $(\beta=.32)$ were significant unique predictors explaining $7 \%$ and $10 \%$ of the variance respectively. The second stage model was significant $(F(6,108)=7.26, p<.001)$ and accounted for an additional and significant $10 \%$ of the variance $\left(F_{\text {change }}(2,108)=7.47, p<.001\right)$. Child SCQ score $(\beta=.28)$, medication status $(\beta=.32)$, and parental mental health status $(\beta=.31)$ were identified as unique 
significant predictors explaining $7 \%, 10 \%$, and $9 \%$ of the variance respectively. The third stage model was also significant $(F(9,105)=5.18, p<.001)$ but did not account for any additional significant variance from the second stage model $\left(F_{\text {change }}(3,105)=\right.$ $.99, p=.39)$. Child SCQ score $(\beta=.34)$, medication status $(\beta=.34)$, and parental mental health status $(\beta=.29)$ remained as unique significant predictors explaining $9 \%, 11 \%$, and $8 \%$ of the variance respectively. The results of the third stage HMR indicated that higher SCQ scores, the child taking prescribed medication, and a parent with a mental health diagnosis predicted higher scores on the Depression subscale of the DBC-P.

Table 4

Hierarchical Multiple Regression Analyses (reporting standardised coefficients; $\beta$ ) for DBC-P Total and Subscale Scores

\begin{tabular}{|c|c|c|c|c|c|}
\hline \multirow[t]{2}{*}{ DBC-P Subscale } & \multirow[t]{2}{*}{ Step } & \multirow[t]{2}{*}{ Predictor } & $\beta$ Step & $\beta$ Step & $\beta$ Step \\
\hline & & & 1 & 2 & 3 \\
\hline \multirow[t]{9}{*}{ TBPS } & \multirow[t]{4}{*}{1} & Age & -.14 & -.18 & -.18 \\
\hline & & Gender & .00 & -.01 & -.02 \\
\hline & & SCQ & $.51 * *$ & $.50 * *$ & $.53 * *$ \\
\hline & & Medication & $.29 * *$ & $.28 * *$ & $.30 * *$ \\
\hline & \multirow[t]{2}{*}{2} & Family Income & & -.11 & -.12 \\
\hline & & Parent Mental Health & & $.22 *$ & $.20 *$ \\
\hline & \multirow[t]{3}{*}{3} & VABS Daily Living Skills & & & .10 \\
\hline & & VABS Communication & & & -.05 \\
\hline & & VABS Socialisation & & & .05 \\
\hline$\Delta R^{2}$ & & & & $.06^{*}$ & .01 \\
\hline$R^{2}$ & & & .37 & .42 & .43 \\
\hline \multirow{4}{*}{$\begin{array}{l}\text { Disruptive } \\
\text { Behaviour }\end{array}$} & \multirow[t]{4}{*}{1} & Age & -05 & -11 & -12 \\
\hline & & Gender & -.02 & -.54 & -.04 \\
\hline & & SCQ & $.23 *$ & $.23 *$ & $.28 *$ \\
\hline & & Medication & $.29 * *$ & $.28 * *$ & $.28 * *$ \\
\hline
\end{tabular}




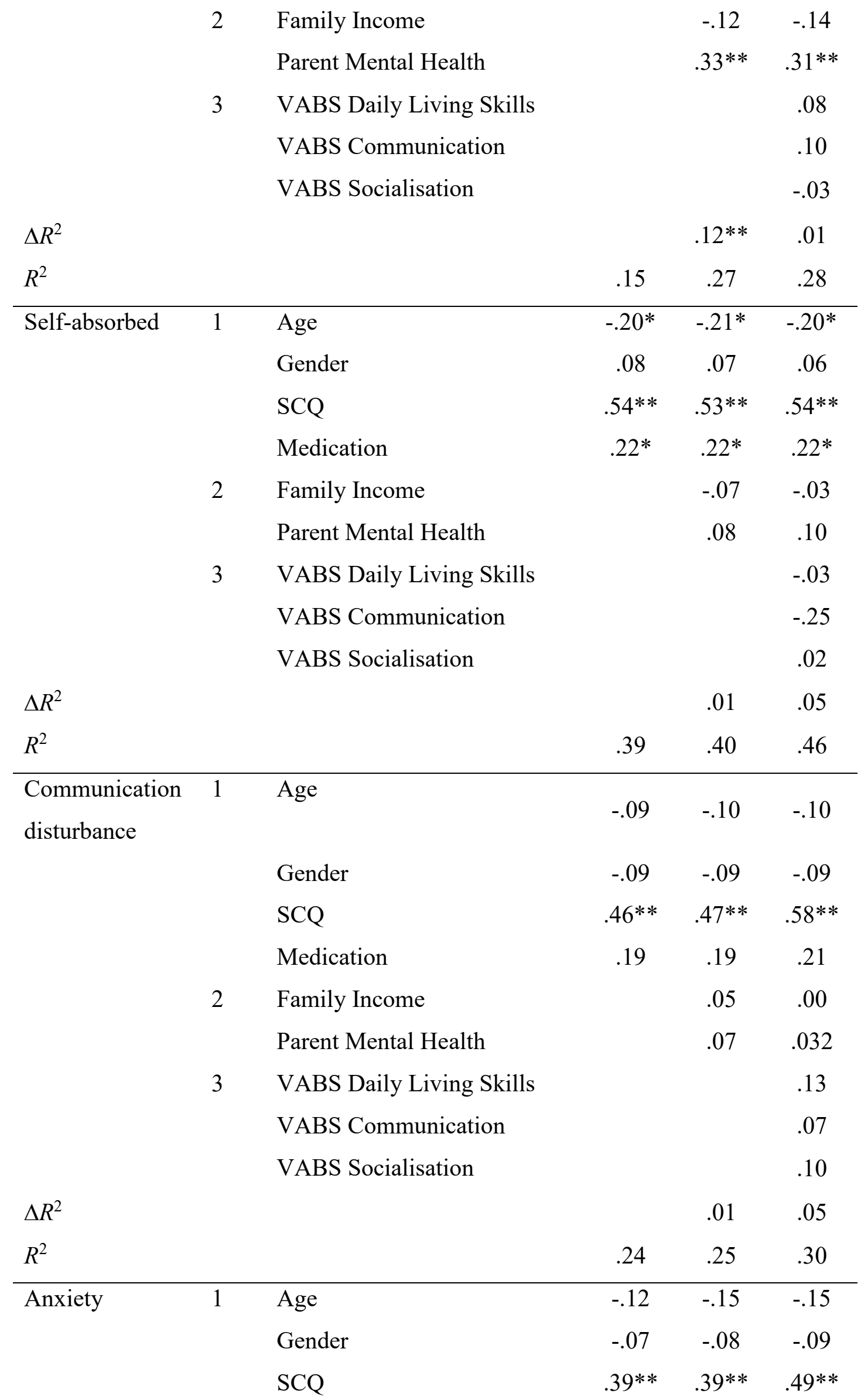




\begin{tabular}{|c|c|c|c|c|c|}
\hline & & Medication & .20 & .20 & .22 \\
\hline & 2 & Family Income & & -.10 & -.15 \\
\hline & & Parent Mental Health & & .15 & .11 \\
\hline & 3 & VABS Daily Living Skills & & & .11 \\
\hline & & VABS Communication & & & .06 \\
\hline & & VABS Socialisation & & & .14 \\
\hline$\Delta R^{2}$ & & & & .03 & .05 \\
\hline$R^{2}$ & & & .20 & .24 & .29 \\
\hline Social Relating & 1 & Age & -.16 & -.20 & $-.21 *$ \\
\hline & & Gender & .08 & .06 & .06 \\
\hline & & SCQ & $.51 * *$ & $.49 * *$ & $.50 * *$ \\
\hline & & Medication & .16 & .14 & .14 \\
\hline & 2 & Family Income & & -.19 & -.19 \\
\hline & & Parent Mental Health & & .19 & .17 \\
\hline & 3 & VABS Daily Living Skills & & & .18 \\
\hline & & VABS Communication & & & .04 \\
\hline & & VABS Socialisation & & & -.15 \\
\hline$\Delta R^{2}$ & & & & $.07 *$ & .02 \\
\hline$R^{2}$ & & & .32 & .39 & .41 \\
\hline Hyperactivity & 1 & Age & .02 & -.01 & -.02 \\
\hline & & Gender & -.03 & -.05 & -.03 \\
\hline & & SCQ & .22 & .20 & .15 \\
\hline & & Medication & .24 & .22 & .20 \\
\hline & 2 & Family Income & & -.16 & -.14 \\
\hline & & Parent Mental Health & & .10 & .12 \\
\hline & 3 & VABS Daily Living Skills & & & -.04 \\
\hline & & VABS Communication & & & .07 \\
\hline & & VABS Socialisation & & & -.15 \\
\hline$\Delta R^{2}$ & & & & .04 & .01 \\
\hline$R^{2}$ & & & .11 & .15 & .16 \\
\hline Depression & 1 & Age & -.05 & -.10 & -.09 \\
\hline & & Gender & -.03 & -.04 & -.06 \\
\hline & & SCQ & $.27 *$ & $.28 * *$ & $.34 * *$ \\
\hline
\end{tabular}




\begin{tabular}{|c|c|c|c|c|c|}
\hline & & Medication & $.32 * *$ & $.32 * *$ & $.34 * *$ \\
\hline & 2 & Family Income & & -.06 & -.09 \\
\hline & & Parent Mental Health & & $.31 * *$ & $.29 * *$ \\
\hline & 3 & VABS Daily Living Skills & & & .10 \\
\hline & & VABS Communication & & & -.08 \\
\hline & & VABS Socialisation & & & .13 \\
\hline$\Delta R^{2}$ & & & & $.10 *$ & .02 \\
\hline$R^{2}$ & & & .19 & .29 & .31 \\
\hline
\end{tabular}

\section{Discussion}

This work extends the work of Chandler et al. (2016) by examining the behavioural and emotional presentation in a sample of 130 young children on the autism spectrum aged $4-5$ years in relation to their level of adaptive functioning and both child and family factors. This is a novel contribution, as although it has been argued that adaptive behaviour is one of the best indicators of level of functioning in individuals on the autism spectrum (Klin et al., 2007), the relative influence of the child's adaptive behaviour on their emotional and behavioural profile for young children on the autism spectrum had not previously been explored. The results highlight that specific child (SCQ score) and family (parental mental health diagnosis) factors were predictive of specific aspects of a child's behavioural and/or emotional profile. When controlling for these child and family factors, child adaptive behaviour was not a significant predictor of a child's behavioural and/or emotional profile as measured by the DBC TBPS or subscale scores. This supports the previous finding that emotional and/or behavioural difficulties can be present in children on the autism spectrum regardless of their ability level (e.g. Chandler et al., 2015; Simonoff et al., 2008) and may be better predicted by autism characteristics than level of functioning. Therefore, support to reduce these difficulties should be available to all children and 
families, regardless of ability. Our findings are in contrast to our hypothesis that adaptive functioning would be a significant independent predictor. However, we found support for hypotheses two and three, which predicted an independent significant impact of child and family factors and the differing of predictors for each DBC-P subscale.

Overall, $79 \%$ of children scored above the clinical cut-off for significant emotional and/or behavioural problems on the DBC-P. Chandler et al. (2016) had a similar finding despite a 12-month difference in age between the two cohorts (average age of 6 years for Chandler et al. versus approximately 5 years in our study). The consistency of this finding across the early years is concerning, especially when considered alongside total population data that suggest challenging behaviour and emotional difficulties are present in young children on the autism spectrum (e.g., Keen, Adams, Simpson, den Houting \& Roberts, 2017; Totsika, Hastings, Emerson, Berridge, \& Lancaster, 2011), persist into childhood (e.g., den Houting, Adams, Roberts, \& Keen, 2018; Totsika et al, 2013), and increase into adolescence (Emerson et al., 2001). These findings therefore highlight the critical importance of intervention efforts in the early years to reduce the risk of, or change the course of, emotional and/or behavioural problems.

A higher score on the SCQ, and parents reporting that their child was taking prescription medication, both made significant unique contributions to children's emotional and behavioural presentation, predicting a higher TPBS overall, as well as higher scores on the Disruptive behaviour, Self-absorbed, Anxiety, and Depression subscales of the DBC. The most commonly reported medication in this sample was melatonin, most frequently used to aid sleep onset. There is a high prevalence of sleep disorders in children on the autism spectrum (Cohen, Conduit, Lockley, Rajaratnam, 
\& Cornish, 2014) and, for many years, it has been recognised that there is an association reported between sleep problems and both emotional and behavioural difficulties (Wiggs \& Stores, 1996). Rzepecka, McKenzie, McClure, and Murphy (2011) also report medication use (the most common of which was also melatonin) and sleep difficulties as predictive of challenging behaviour in their sample of 187 individuals with a diagnosis on the autism spectrum and/or ID. These findings are interesting given that melatonin has been shown to decrease daytime challenging behaviour (Braam et al., 2010; van Maanen, Meijer, Smits, \& Oort, 2011) and may suggest an interaction between the emotional and/or behavioural presentation of the child and the impact that these behaviours have on caregivers seeking input from additional professionals (Adams, Handley, et al., 2018).

The SCQ score alone was found to predict higher levels of communication disturbance in children, while the SCQ score and child age together predicted scores on the social relating subscale. It is worth noting that the addition of the VABS domains did not add any significant additional variance to any of the models and none of the subdomains of the VABS were significant unique predictors in any of the regression models despite adaptive behaviours, particularly communication skills, being recognised as a strong predictor of outcomes more broadly (Klin et al., 2007). While family income was not found to predict any aspects of behaviour in the current study, parent mental health emerged as a significant predictor of TPBS, Disruptive Behaviour subscale, and Depression subscale, making a unique contribution over and above their SCQ score and medication use, explaining between $3-9 \%$ of the variance in scores. Although causation cannot be inferred, these findings are aligned with the wealth of research documenting the bidirectional association between increased mental health problems in parents of children with behaviours that challenge 
(e.g.,Woodman, Mawdsley, \& Hauser-Cram, 2015). The increased stress and mental health difficulties (Adams, Hasting et al., 2018; Estes et al., 2013; Falk, Norris, \& Quinn, 2014; Firth \& Dryer, 2013; Paynter, Riley, Beamish, Davies, \& Milford, 2013) and poorer quality of life (Adams, Clark \& Simpson, 2019) experienced by parents of children on the autism spectrum have been well documented.

Salomone et al. (2018) found that behavioural difficulties of school age children on the autism spectrum placed additional strain on the mental health of their parents, predicting poorer mental health outcomes. If these behaviours remain unsupported, a bidirectional risk is posed of worsening the mental health and wellbeing of their parents, which - based on the current findings - will contribute to more emotional and behavioural difficulties in their children. Further efforts are warranted to support the positive mental health and development of coping strategies in parents raising children on the autism spectrum, especially those with behavioural or emotional difficulties (Adams, Rose, Jackson, Karakatstani, \& Oliver, 2018). When considering how to best support the entire family unit, focusing solely on early intervention to manage the emotional and behavioural problems of children on the spectrum may not be sufficient. Interventions taking a family-centred approach by supporting the whole system, including the child, their parents, and the broader family, may result in more positive outcomes ( $<$ removed for blind review $>$ ) by promoting better mental health for parents to minimise emotional and behavioural difficulties in their children on the autism spectrum (Crnic, Neece, McIntyre, Blacher, \& Baker, 2017).

\section{Limitations}

There are a number of limitations which need to be recognised within the present study. First, although there is a strong rationale for the use of the adaptive 
behaviour measure, future research using both adaptive and intellectual functioning would be of value to ascertain whether differences are apparent in verified ID subgroups and whether this is a meaningful distinction. There is also limited information on additional or co-morbid diagnoses which would warrant exploration with regards to the impact on behavioural profiles. Third, as this group used a restricted age range ( $4-5$ years), it is unclear how behaviour may manifest, change, or develop in older children. Although evidence suggests the challenging behaviours may increase with age (e.g., Emerson et al., 2001), there is little research describing how a broader range of "non-challenging" behavioural difficulties develop with age, such as those measured on the DBC. This would be particularly pertinent given the recent focus on considering individual topographies of behaviour rather than “challenging behaviours" as a whole (e.g. Adams, Clarke, et al., 2018). Thus, future research with a range of ages, using cohort, longitudinal, or sequential designs, would be of value to map the developmental trajectory of a range of behaviours and explorations for the trajectory of specific topographies of challenging behaviour. As this study was based on an online survey, there was no independent verification of autism diagnosis. Although SCQ scores and community diagnostic reports were used to confirm diagnosis, the diagnostic assessment process detailed within the diagnostic reports varied across children. Finally, it is important to recognise that there are numerous additional variables which may influence both the emotional and/or behavioural presentation and their adaptive behaviour which were not measured or included in these models.

\section{Future Directions}

Future studies exploring the impact of IQ and adaptive behaviour within the sample would extend our understanding of the impact of functioning on emotional 
and behavioural presentation in young children on the autism spectrum. Further work exploring the $\mathrm{DBC}$ with a range of ages and abilities may also allow for the identification of subgroups based on behavioural profiles and/or adaptive behaviour that may show different trajectories of response to intervention, which would help to address the vexed question of "what works for whom?". This is of clear importance given the high rates of challenging behaviour in this population and the resultant impact such behaviours are likely to have not only on the child, but also on their school, family, and community. Further understanding the behavioural profile of children on the autism spectrum and potential subtypes is thus a ripe area for continued research to better support children, families, and schools to achieve optimal outcomes.

Conflict of Interest: None of the authors have any conflicts of interest relevant to this article to disclose.

Funding: The authors acknowledge the financial support of the Cooperative Research Centre for Living with Autism (Autism CRC), established and supported under the Australian Government's Cooperative Research Centres Program (project $<$ removed for blind review>). 


\section{References}

Achenbach, T., \& Edlbrock, C. (1983). Manual for the child behavior checklist and revised child behavior profile. Burlington: Universtiy of Vermont Associates in Psychiatry.

Adams, D., Clark, M. \& Simpson, (2019). The relationship between child anxiety and the quality of life of children, and parents of children, on the autism spectrum. Journal of Autism and Developmental Disabilities, ePub ahead of print. Doi: $10.1007 / \mathrm{s} 10803-019-03932-2$.

Adams, D., Clarke, S., Griffith, G., Howlin, P., Moss, J., Petty, J., . . Oliver, C. (2018). Mental health and well-being in mothers of children with rare genetic syndromes showing chronic challenging behavior: A cross-sectional and longitudinal study. American Journal on Intellectual and Developmental Disabilities, 123(3), 241-253. doi:10.1352/1944-7558-123.3.241

Adams, D., Handley, L., Simkiss, D., Walls, E., Jones, A., Knapp, M., . . Oliver, C. (2018). Service use and access in young children with an intellectual disability or global developmental delay: Associations with challenging behaviour. Journal of Intellectual \& Developmental Disability, 43(2), 232-241. doi:10.3109/13668250.2016.1238448

Adams, D., Hastings, R., Alston-Knox, A., Cianfaglione, R., Eden, K., Felce, D., Griffith, G., Moss, J, Stinton, C. \& Oliver, C. (2018). Using Bayesian methodology to explore the profile of mental health and well-being in 646 mothers of children with 13 rare genetic syndromes in relation to mothers of children with autism. Orphanet Journal of Rare Diseases, 13, 185. doi: 10.1186/s13023-018-0924-1 
Adams, D., Rose, J., Jackson, N., Karakatsani, E., \& Oliver, C. (2018). Coping strategies in mothers of children with intellectual disabilities showing multiple forms of challenging behaviour: associations with maternal mental health. Behavioural and cognitive psychotherapy, 46(3), 257-275.

Akshoomoff, N. (2006). Use of the Mullen Scales of Early Learning for the assessment of young children with Autism Spectrum Disorders. Child Neuropsychology, 12(4-5), 269-277. doi:10.1080/09297040500473714

Baghdadli, A., Pascal, C., Grisi, S., \& Aussilloux, C. (2003). Risk factors for selfinjurious behaviours among 222 young children with autistic disorders. Journal of Intellectual Disability Research 47(8) 622-627.

Braam, W., Didden, R., Maas, A. P., Korzilius, H., Smits, M. G., \& Curfs, L. M. (2010). Melatonin decreases daytime challenging behaviour in persons with intellectual disability and chronic insomnia. Journal of Intellectual Disability Research, 54(1), 52-59. doi:10.1111/j.1365-2788.2009.01223.x

Brereton, A., Tonge, B., \& Einfeld, S. L. (2006). Psychopathology in children and adolescents with autism compared to young people with intellectual disability. Journal of Autism and Developmental Disorders, 36, 863-870. doi:10.1007/s10803-006-0125-y

Chandler, S., Howlin, P., Simonoff, E., O'Sullivan, T., Tseng, E., Kennedy, J., . . . Baird, G. (2016). Emotional and behavioural problems in young children with autism spectrum disorder. Developmental Medicine and Child Neurology, 58, 202-208. doi:10.1111/dmen. 12830

Chesnut, S. R., Wei, T., Barnard-Brak, L., \& Richman, D. M. (2017). A meta-analysis of the social communication questionnaire: Screening for autism spectrum disorder. Autism, 21(8), 920-928. doi:10.1177/1362361316660065 
Close, H. A., Lee, L. C., Kaufmann, C. N., \& Zimmerman, A. W. (2012). Cooccurring conditions and change in diagnosis in autism spectrum disorders. Pediatrics, 129(2), e305-316. doi:10.1542/peds.2011-1717

Cohen, S., Conduit, R., Lockley, S. W., Rajaratnam, S. M. W., \& Cornish, K. M. (2014). The relationship between sleep and behavior in autism spectrum disorder (ASD): A review. Journal of Neurodevelopmental Disorders, 6(44). doi:Artn 4410.1186/1866-1955-6-44

Crnic, K. A., Neece, C. L., McIntyre, L. L., Blacher, J., \& Baker, B. L. (2017). Intellectual disability and developmental risk: Promoting intervention to improve child and family well-being. Child development, 88(2), 436-445.

den Houting, J., Adams, D., Roberts, J., \& Keen, D. (2018). Exploring anxiety symptomatology in school-aged autistic children using an autism-specific assessment. Research in Autism Spectrum Disorders, 50, 73-82. doi:10.1016/j.rasd.2018.03.005

Eaves, L. C., Wingert, H. D., Ho, H. H., \& Mickelson, E. C. (2006). Screening for autism spectrum disorders with the social communication questionnaire. Journal of Developmental and Behavioral Pediatrics, 27(2). doi:10.1097/00004703-200604002-00007

Einfeld, S. L., \& Tonge, B. J. (1995). The Developmental Behavior Checklist: The development and validation of an instrument to assess behavioral and emotional disturbance in children and adolescents with mental retardation. Journal of Autism and Developmental Disorders, 25(2), 81-104. doi:10.1007/bf02178498

Emerson, E., Kiernan, C., Alborz, A., Reeves, D., Mason, H., Swarbrick, R., . . . Hatton, C. (2001). The prevalence of challenging behaviors: A total 
population study. Research in Developmental Disabilities, 22(1), 77-93. doi:10.1016/S0891-4222(00)00061-5

Estes, A., Olson, E., Sullivan, K., Greenson, J., Winter, J., Dawson, G., \& Munson, J. (2013). Parenting-related stress and psychological distress in mothers of toddlers with autism spectrum disorders. Brain and Development, 35(2), 133138. doi:10.1016/j.braindev.2012.10.004

Falk, N., Norris, K., \& Quinn, M. (2014). The factors predicting stress, anxiety and depression in the parents of children with autism. Journal of Autism and Developmental Disorders, 44, 3185-3203. doi:10.1007/s10803-014-2189-4

Firth, I., \& Dryer, R. (2013). The predictors of distress in parents of children with autism spectrum disorder. Journal of Intellectual and Developmental Disability, 38(2), 163-171. doi:10.3109/13668250.2013.773964

Goodman, R. (1997). The Strengths and Difficulties Questionnaire: A research note. Journal of Child Psychology and Psychiatry, 38(5), 581-586.

Herring, S., Gray, K., Taffe, J., Tonge, B., Sweeney, D., \& Einfeld, S. (2006). Behaviour and emotional problems in toddlers with pervasive developmental disorders and developmental delay: Associations with parental mental health and family functioning. Journal of Intellectual Disability Research, 50, 874882. doi:10.1111/j.1365-2788.2006.00904.x

Jellett, R., Wood, C., Giallo, R., \& Seymour, M. (2015). Family functioning and behaviour problems in children with autism spectrum disorders: The mediating role of parent mental health. Clinical Psychologist, 19, 39-48. doi: $10.1111 /$ cp. 12047 
Keen, D., Adams, D., Simpson, K., den Houting, J., \& Roberts, J. (2017). Anxietyrelated symptomatology in young children on the autism spectrum. Autism, ePub ahead of print: doi:10.1177/1362361317734692

Kenworthy, L., Case, L., Harms, M. B., Martin, A., \& Wallace, G. L. (2010). Adaptive behavior ratings correlate with symptomatology and IQ among individuals with high-functioning autism spectrum disorders. Journal of autism and developmental disorders, 40(4), 416-423.

Klin, A., Saulnier, C. A., Sparrow, S. S., Cicchetti, D. V., Volkmar, F. R., \& Lord, C. (2007). Social and communication abilities and disabilities in higher functioning individuals with autism spectrum disorders: The Vineland and the ADOS. Journal of Autism and Developmental Disorders, 37, 748-759. doi:10.1007/s10803-006-0229-4

Kurzius-Spencer, M., Pettygrove, S., Christensen, D., Pedersen, A. L., Cunniff, C., Meaney, F. J., . . Rice, S. (2018). Behavioral problems in children with autism spectrum disorder with and without co-occurring intellectual disability. Research in Autism Spectrum Disorders, 56, 61-71. doi:10.1016/j.rasd.2018.09.002

Lee, L.-C., David, A. B., Rusyniak, J., Landa, R., \& Newschaffer, C. (2007). Performance of the Social Communication Questionnaire in children receiving preschool special education services. Research in Autism Spectrum Disorders, 1, 126-138. doi:10.1016/j.rasd.2006.08.004

Liss, M., Harel, B., Fein, D., Allen, D., Dunn, M., Feinstein, C., ... \& Rapin, I. (2001). Predictors and correlates of adaptive functioning in children with developmental disorders. Journal of autism and developmental disorders, 31, 219-230. 
Maskey, M., Warnell, F., Parr, J., Le Couteur, A., and McConachie, H. (2013). Emotional and behavioural problems in children with autism spectrum disorder." Journal of autism and developmental disorders 43(4) 851-859.

Mayes, S. D., Calhoun, S. L., Murray, M. J., Ahuja, M., \& Smith, L. A. (2011). Anxiety, depression, and irritability in children with autism relative to other neuropsychiatric disorders and typical development. Research in Autism Spectrum Disorders, 5(1), 474-485. doi:http://dx.doi.org/10.1016/j.rasd.2010.06.012

McClintock, K., Hall, S., and Oliver, C. (2003). Risk markers associated with challenging behaviours in people with intellectual disabilities: a meta-analytic study. Journal of Intellectual Disability Research 47(6) 405-416.

Nader, A. M., Courchesne, V., Dawson, M., \& Soulieres, I. (2016). Does WISC-IV underestimate the intelligence of autistic children? Journal of Autism and Developmental Disorders, 46(5), 1582-1589. doi:10.1007/s10803-014-2270-z

Park, C. J., Yelland, G. W., Taffe, J. R., \& Gray, K. M. (2012). Brief report: The relationship between language skills, adaptive behavior, and emotional and behavior problems in pre-schoolers with autism. Journal of Autism and Developmental Disorders, 42(12), 2761-2766.

Paynter, J., Riley, E., Beamish, W., Davies, M., \& Milford, T. (2013). The double ABCX model of family adaptation in families of a child with an autism spectrum disorder attending an Australian early intervention service. Research in Autism Spectrum Disorders, 7(10), 1183-1195. doi:http://dx.doi.org/10.1016/j.rasd.2013.07.006

Perneger, T. V. (1998). What's wrong with Bonferroni adjustments. Bmj, 316(7139), $1236-1238$. 
Reynolds, C. R., \& Kamphaus, R. W. (2015). Behavior assessment system for children (3rd ed.). MN: AGS Publishing.

Rattaz, C., Michelon, C., Munir, K., Baghdadli, A. (2018). Challenging behaviours at early adulthood in autism spectrum disorders: topography, risk factors and evolution. Journal of Intellect Disability Research 62(7) 637-649.

Rutter, M., Bailey, A., \& Lord, C. (2003). Social Communication Questionnaire. Los Angeles, CA: Western Psychological Services.

Rzepecka, H., McKenzie, K., McClure, I., \& Murphy, S. (2011). Sleep, anxiety and challenging behaviour in children with intellectual disability and/or autism spectrum disorder. Research in Developmental Disabilities, 32(6), 2758-2766. doi:10.1016/j.ridd.2011.05.034

Salomone, E., Leadbitter, K., Aldred, C., Barrett, B., Byford, S., Charman, T., . . . Consortium, P. (2018). The association between child and family characteristics and the mental health and wellbeing of caregivers of children with autism in mid-childhood. Journal of Autism and Developmental Disorders, 48(4), 1189-1198. doi:10.1007/s10803-017-3392-х

Sharma, A. and S. R. Shaw (2012). Efficacy of risperidone in managing maladaptive behaviors for children with autistic spectrum disorder: a meta-analysis. Journal Pediatric Health Care 26(4) 291-299.

Sikora, D. M., Vora, P., Coury, D. L., \& Rosenberg, D. (2012). Attentiondeficit/hyperactivity disorder symptoms, adaptive functioning, and quality of life in children with autism spectrum disorder. Pediatrics, 130 Suppl 2, S9197. doi:10.1542/peds.2012-0900G

Simonoff, E., Pickles, A., Charman, T., Chandler, S., Loucas, T., \& Baird, G. (2008). Psychiatric disorders in children with autism spectrum disorders: prevalence, 
comorbidity, and associated factors in a population-derived sample. Journal of the American Academy of Child \& Adolescent Psychiatry, 47(8), 921-929.

Smith, I. M. (2016). Expanding our understanding of behavioural difficulties associated with autism spectrum disorder. Developmental Medicine \& Child Neurology, 58(2), 121-122. doi:10.1111/dmcn.12855

Soke, G. N., Maenner, M. J., Christensen, D., Kurzius-Spencer, M., \& Schieve, L. A. (2018). Prevalence of co-occurring medical and behavioral conditions/symptoms among 4- and 8-year-old children with autism spectrum disorder in selected areas of the United States in 2010. Journal of Autism and Developmental Disorders, 48(8), 2663-2676. doi:10.1007/s10803-018-3521-1

Sparrow, S. S., Cicchetti, D. V., \& Balla, D. A. (2005). Vineland Adaptive Behavior Scales 2nd Edition (2nd ed.). Minneapolis: NCS Pearson, Inc.

Taylor, L. J. (2016). Psychopharmacologic intervention for adults with autism spectrum disorder: a systematic literature review. Research in Autism Spectrum Disorders, 25, 58-75.

Tonge, B., Brereton, A., Kiomall, M., Mackinnon, A., King, N., \& Rinehart, N. (2006). Effects on parental mental health of an education and skills training program for parents of young children with autism: A randomized controlled trial. Journal of the American Academy of Child and Adolescent Psychiatry, 45(5), 561-569. doi:10.1097/01.chi.0000205701.48324.26

Totsika, V., Hastings, R.P., Emerson, E., Lancaster, G.A., \& Berridge, D.M. (2011). A population-based investigation of behavioural and emotional problems and maternal mental health: associations with autism spectrum disorder and intellectual disability. Journal of Child Psychology and Psychiatry 52(1) 9199. 
Totsika V., Hastings R.P., Emerson E., Berridge D.M., Lancaster G.A. (2011).

Behavior Problems at 5 Years of Age and Maternal Mental Health in Autism and Intellectual Disability. Journal of Abnormal Child Psychology, 39, 11371147.

Totsika, V., Hastings, R. P., Emerson, E., Lancaster, G. A., Berridge, D. M., \& Vagenas, D. (2013). Is there a bidirectional relationship between maternal well-being and child behavior problems in autism spectrum disorders? Longitudinal analysis of a population-defined sample of young children. Autism Research, 6(3), 201-211.

Tureck, K., Matson, J. L., Turygin, N., \& Macmillan, K. (2013). Rates of psychotropic medication use in children with ASD compared to presence and severity of problem behaviors. Research in Autism Spectrum Disorders, 7(11), $1377-1382$.

van Maanen, A., Meijer, A. M., Smits, M. G., \& Oort, F. J. (2011). Termination of short term melatonin treatment in children with delayed Dim Light Melatonin Onset: Effects on sleep, health, behavior problems, and parenting stress. Sleep Medicine, 12(9), 875-879. doi:10.1016/j.sleep.2011.03.019

Vohra, R., Madhavan, S., \& Sambamoorthi, U. (2016). Emergency department use among adults with autism spectrum disorders (ASD). Journal of Autism and Developmental Disorders, 46(4), 1441-1454. doi:10.1007/s10803-015-2692-2

Wiggs, L., \& Stores, G. (1996). Severe sleep disturbance and daytime challenging behaviour in children with severe learning disabilities. Journal of Intellectual Disability Research, 40(6), 518-528. 
Williams, D. L., Siegel, M., \& Mazefsky, C. A. (2018). Problem behaviors in autism spectrum disorder: Association with verbal ability and adapting/coping skills. Journal of autism and developmental disorders, 48(11), 3668-3677.

Woodman, A. C., Mawdsley, H. P., \& Hauser-Cram, P. (2015). Parenting stress and child behavior problems within families of children with developmental disabilities: Transactional relations across 15 years. Research in Developmental Disabilities, 36C, 264-276. doi:10.1016/j.ridd.2014.10.011

Wright, B., Sims, D., Smart, S., Alwazeer, A., Alderson-Day, B., Allgar, V., . . . Miles, J. (2011). Melatonin versus placebo in children with autism spectrum conditions and severe sleep problems not amenable to behaviour management strategies: A randomised controlled crossover trial. Journal of Autism and Developmental Disorders, 41(2), 175-184. doi:10.1007/s10803-010-1036-5

Yang, S., Paynter, J. M., \& Gilmore, L. (2016). Vineland adaptive behavior scales: II profile of young children with autism spectrum disorder. Journal of autism and developmental disorders, 46(1), 64-73. 